\title{
Distribución de Festuca patula Desf. en la Península Ibérica; ecología y tamaños poblacionales de sus poblaciones en Las Villuercas y Los Montes de Toledo (CW de España)
}

\author{
Juan A. Calleja Alarcón ${ }^{1}$
}

\begin{abstract}
Resumen: Calleja Alarcón, J.A. . 2011. Distribución de Festuca patula Desf. en la Península Ibérica; ecología y tamaños poblacionales de sus poblaciones en Las Villuercas y Los Montes de Toledo. Bot. Complut. 35: 39-48.

Se presentan datos sobre el tamaño de las poblaciones y las preferencias de hábitat de Festuca patula Desf. en Las Villuercas y Los Montes de Toledo (CW de España). Se amplia la información corológica para la península con 21 localidades nuevas: una en Sierra Nevada (Granada) y el resto en Las Villuercas y Los Montes de Toledo (Cáceres, Toledo y Ciudad Real). Las poblaciones de estos dos últimos territorios habitan en distintas condiciones ambientales. Se encuentran entre $10 s 300$ y $1.000 \mathrm{~m}$ de altitud, instaladas en sustratos metamórficos, creciendo en el sotobosque de frondosas (quejigos, alcornoques, encinas, castaños, etc.) y en los claros de matorrales en laderas rocosas que, en numerosas ocasiones, limitan con formaciones riparias (caracterizadas por alisos, fresnos, sauces, etc.). La mayoría de las poblaciones están integradas por menos de 100 ejemplares y solamente dos alcanzan o superan los 1.000 individuos. El tamaño poblacional parece ser mayor en enclaves con suelo relativamente desarrollado, en ambientes forestales sombríos.
\end{abstract}

Palabras clave: corología, autoecología, flora, Península Ibérica, censo poblacional, Festuca patula.

Abstract: Calleja Alarcón, J.A.. 2011. Distribution of Festuca patula Desf. in the Iberian Peninsula; ecology and population sizes of its populations in Las Villuercas and Los Montes de Toledo. Bot. Complut. 35: 39-48.

Population sizes and habitat types of Festuca patula Desf. are reported for Las Villuercas and Los Montes de Toledo regions (CW Spain). The distribution range of Festuca patula in the Iberian Peninsula is increased with 21 new records: one for Sierra Nevada (Granada, S Spain) and 20 for The Villuercas and Los Montes de Toledo (Cáceres, Toledo and Ciudad Real). The individuals of the latter areas grow under different environmental conditions. They live on rocky or relatively well-developed soils derived from metamorphic rocks, ranging from 300 to $900 \mathrm{~m}$ above sea level. They usually grow in temperate caducifolious and marcescent forests but also colonize open scrubs on rocky slopes, often bordering riparian stands. Most of the populations contain less than 100 individuals; only one population has more than 1,000. The population sizes are apparently larger on shady wooded sites with relatively well-developed soils than in rocky sites.

Key words: chorology, autoecology, flora, Iberian Peninsula, population censuses, Festuca patula.

\section{INTRODUCCION}

Festuca patula Desf., es una gramínea perenne iberonorteafricana (Devesa 1992). La planta ha sido mayoritariamente nombrada Festuca triflora Desf. (Mueller \& Catalán 2006, Catalán 2009), sin embargo una revisión nomenclatural reciente reivindica como nombre prioritario Festuca patula (Mueller \& Catalán 2006). La especie había sido citada fundamentalmente en la mitad meridional de la Península Ibérica y en especial en las Cordilleras Béticas y en Sierra Morena (Cebolla \& Rivas Ponce
1990, García Río 1999). De manera mucho más dispersa, se ha mencionado su presencia en Extremadura (Devesa 1995) y se ha citado en Los Montes de Toledo (Ciudad Real y Toledo) (Cebolla \& Rivas 1990), y en localidades septentrionales ibéricas más alejadas en el Sistema Central (Guadalajara, Mayor 1975) y en el Caurel (Lugo, Merino 1907). En general, y atendiendo a los escuetos comentarios incluidos en pliegos, floras y catálogos, parece tratarse de una especie poco abundante 0 rara (Aparicio \& Silvestre 1987, Devesa 1995, García 2004, Catalán 2009). Es indiferente edáfica y coloniza diferentes hábitats pues hay

${ }^{1}$ C1/339. Botánica, Dpto. Biología Animal, Vegetal y Ecología, Universidad Autónoma de Barcelona, E-08193 Bellaterra, Barcelona, España.juan.calleja@gmail.com; juanantonio.calleja@uab.cat

Recibido: 21 diciembre 2010. Aceptado: 14 febrero 2011 
autores que describen su preferencia por sotobosques sombríos montanos (Aparicio \& Silvestre 1987, Devesa 1995) y por suelos profundos en medios forestales (García, 2004), mientras que otros señalan su presencia en ambientes expuestos, tales como roquedos, lapiaces y herbazales sobre suelos pedregosos (Cebolla \& Rivas 1990, Serra et al. 2000).

En el presente trabajo se aportan nuevos datos sobre la distribución geográfica de F. patula en la Península Ibérica. Complementariamente, se analizan las características del hábitat y los tamaños poblacionales de esta especie en las sierras de Las Villuercas (Cáceres) y Los Montes de Toledo (Toledo y Ciudad Real) y se realiza una síntesis comparada de sus preferencias ecológicas en estos sistemas montañosos con respecto al resto de las localidades ibéricas. Los objetivos específicos del estudio son: i- actualizar la información corológica de F. patula a escala peninsular, contrastando citas previas y aportando localidades nuevas para Los Montes de Toledo y Las Villuercas; ii- describir las condiciones ambientales en las que vive la planta en las zonas de estudio, comparándolas con las de otras poblaciones ibéricas; iii- analizar los tamaños poblacionales obtenidos en los censos desarrollados en las zonas de estudio; y, iv- analizar si la insolación y el desarrollo del suelo junto con la altitud pueden condicionar su abundancia.

\section{MATERIALES Y MÉTODOS}

Área de estudio. Las sierras de Las Villuercas y Los Montes de Toledo son sistemas montañosos de 3.200 y $5.700 \mathrm{~km}^{2}$ respectivamente situados en el centro de la Meseta meridional de la Península Ibérica (Muñoz \& Sanz 1995). A grandes rasgos, se componen de un conjunto intrincado de cortas alineaciones rocosas que componen un fragmentado relieve que separa las cuencas del río Tajo y el río Guadiana. La altitud media es de $700 \mathrm{~m}$ y las cotas máximas son $1.595 \mathrm{~m}$ en Las Villuercas, y $1.447 \mathrm{~m}$ en Los Montes de Toledo. Predominan los materiales precámbricos y paleozoicos de naturaleza metamórfica, principalmente cuarcitas y pizarras. Los materiales sedimentarios son muy escasos y solo destacan las rañas en la periferia de ambos sistemas montañosos (Muñoz \& Sanz 1995). El clima regional es mediterráneo continental (Font 1983) con unas temperaturas medias de $13-18^{\circ} \mathrm{C}$ y unas precipitaciones que oscilan entre los 500 y $1.200 \mathrm{~mm}$ anuales (http://sig.marm.es/siga). El tapiz vegetal es un mosaico de bosques y formaciones arborescentes dominados por quercíneas perennes (Quercus ilex subsp. ballota y $Q$. suber) y marcescentes (Q. pyrenaica y $Q$. faginea subsp. broteroi). Albergan un cortejo rico en madroños (Arbutus unedo), durillos (Viburnum tinus), olivillas (Phillyrea sp. pl.), brezos (Erica sp. pl.) y jaras (Cistus sp. pl.) (Ladero 1987,
Costa et al. 1997, Pulido et al. 2007), que en ocasiones dominan extensas superficies. Por otra parte, las plantaciones de $P i$ nus pinaster son localmente muy abundantes. En las riberas normalmente aparecen las alisedas y fresnedas y, además, son relativamente frecuentes las formaciones mixtas en las que participan, junto con el aliso (Alnus glutinosa) y el fresno (Fraxinus angustifolia), otros arbolillos como los arraclanes (Frangula alnus), sauces (Salix sp. pl.), loros (Prunus lusitanica) y acebos (Ilex aquifolium) (Costa et al. 1997, Herrero et al. 2003, Pulido et al. 2007).

Toma de datos. La localización de las poblaciones de Festuca patula en la Península Ibérica y en especial en las sierras de Las Villuercas y en Los Montes de Toledo se llevó a cabo a partir de la información publicada (Merino 1907, Rivas-Goday \& Rivas-Martínez 1969, Mayor 1975, Aparicio \& Silvestre 1987, Molero \& Pérez-Raya 1987, Cebolla \& Ponce 1990, 1993a, 1993b, Fernández \& Montabés 1995, De la Torre et al. 1996, Fernández \& Fernández 1996, García 1999, 2004, 2006, Cabezudo et al. 2005, Serra et al. 2000, Llamas et al. 2002), los datos descritos en pliegos de herbario accesibles desde diferentes bases de datos (http://www.anthos.es; http://www.gbif.es) o mediante consultas a los responsables de los herbarios (SEV, LOU) 0 en pliegos directamente consultados (MA, BC); además, se realizaron exhaustivas prospecciones de campo en ambos sistemas montañosos. En Las Villuercas se prospectaron las cuencas de los ríos Fresnedoso, Gualija, Almonte e Ibor (tributarios del río Tajo) y los ríos Guadarranque, Ruecas y Guadalupejo (tributarios del Guadiana), y en Los Montes de Toledo las de los ríos Gévalo y Pusa (afluentes del río Tajo) y el río Estena (afluente del Guadiana).

Se consideró una unidad de población al conjunto de ejemplares de $F$. patula que distaban entre sí menos de un km. Para cada población se contaron el número de individuos y se registraron los datos de las siguientes variables: altitud, litología, tipo de suelo, insolación y tipo de vegetación dominante. Los datos de altitud de cada población fueron los de la cota en la que se localizaba el mayor número de individuos de la misma. La litología se registró mediante el reconocimiento directo en campo 0 tras la consulta de mapas geológicos (http://www.igme.es). Para la tipificación del suelo se evaluó visualmente la existencia de los horizontes básicos en los que se puede estructurar un perfil edáfico: A: horizonte orgánico, B: horizonte de acumulación; C: roca madre meteorizada y RC: roca madre poco alterada. El grado de insolación se clasificó en dos categorías: soleado (insolación directa sobre más del 50\% de los individuos de la población) y umbroso (ausencia de insolación directa en más del $50 \%$ de los individuos). En todos los casos, la sombra está generada por la presencia de árboles. La vegetación dominante se agrupó en 10 tipos básicos (la mayoría descritos en Costa et al. 1997): pinsapar (bosques de Abies pinsapo), alcornocal-quejigar (bosques dominados o codominados por Quercus suber y Q. faginea subsp. broteroi), melojar-castañar (bosques dominados o codominados por Quercus pyrenaica o Castanea sativa), encinar (bosques de Quercus ilex subsp. ballota), bosque edafohigrófilo (alisedas, fresnedas, saucedas o loreras), encinar-bosque edafohigrófilo, matorral (formaciones arbustivas 
dominadas o codominadas por Arbutus, Cistus, Erica), matorralbosque edafohigrófilo, herbazales (comunidades dominadas por herbáceas perennes, bianuales y anuales), roquedos (áreas rocosas con escasa cobertura vegetal). Adicionalmente se tomaron datos de las precipitaciones y de las temperaturas medias anuales de 36 estaciones meteorológicas (http://sig.marm.es/siga) situadas en las proximidades de las poblaciones ibéricas de Festuca patula (Anexo II).

Tratamiento y análisis de datos. Con la información corológica obtenida en el trabajo de campo y tras un análisis de los datos disponibles en la bibliografía se elaboró un mapa de distribución de $F$. patula en la península ibérica, en el que se indicó su presencia en cuadrículas UTM 10 x $10 \mathrm{~km}$. Los datos ambientales se emplearon para realizar un análisis comparativo entre los parámetros ambientales que afectan a las poblaciones de Los Montes de Toledo y Las Villuercas y los que afectan a las poblaciones del resto de la Península Ibérica. Además, para las poblaciones de Las Villuercas y Los Montes de Toledo junto con una población del Parque Nacional de Monfragüe (próximo a Las Villuercas) en Cáceres, se estimó la influencia de la altitud, del tipo de suelo y del grado de insolación en el número de individuos de cada población. Para ello se empleó una regresión linear múltiple tipo GLM (General Linear Model) con el programa STATISTICA 6.0 (www.statsoft.com). En este análisis el grado de insolación y el tipo de suelo fueron considerados variables explicativas cualitativas y la altitud fue tratada como variable explicativa continua. De manera previa, se transformó la variable dependiente $N$ (número de individuos) mediante la función logarítmica con objeto de cumplir las premisas del análisis (www.statsoft.com).

\section{RESULTADOS}

Corología de Festuca patula en la Península Ibérica. Las nuevas prospecciones en el CW peninsular aportaron 21 nuevas poblaciones localizadas en 11 cuadrículas UTM $10 \times 10$ km nuevas: seis para Las Villuercas (incluyendo Monfragüe) en Cáceres, cinco para Los Montes de Toledo (Ciudad Real, Toledo) y una para Sierra Nevada (Granada) (Fig. 1, Tabla 1). Todas estas nuevas localidades junto con la revisión crítica de la bibliografía y la consulta en herbarios permitieron definir con mayor precisión el área de distribución geográfica de F. patula en la Península Ibérica con un total de 88 localidades (Anexo I) repartidas en 63 cuadrículas UTM $10 \times 10 \mathrm{~km}$ (Fig. 1).

La mayor parte de las localidades se encuentran en los sistemas montañosos de la mitad sur de la península; especialmente en las Cordilleras Béticas, Sierra Morena, Montes de Toledo y Las Villuercas, dentro de los límites de Alicante (3 localidades), Albacete (1), Almería (2), Badajoz (1), Cádiz (6), Cáceres (16), Córdoba (7), Ciudad Real (14), Granada (6), Jaén (20), Málaga (6), Sevilla (1),

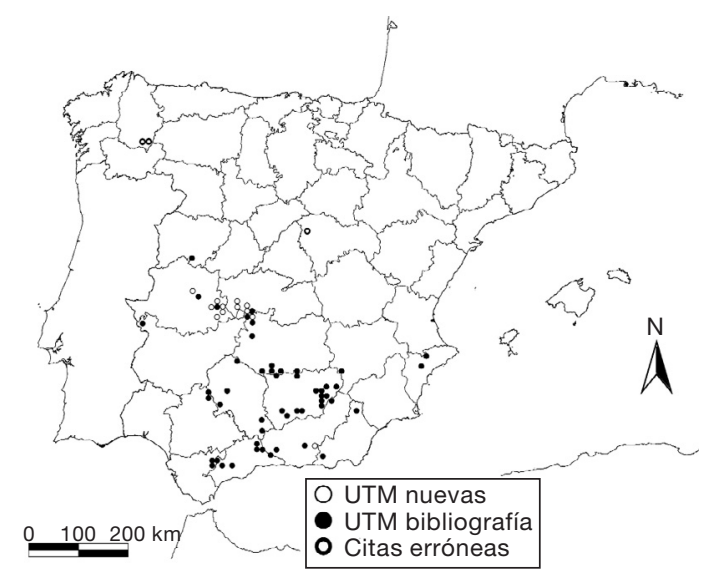

Fig. 1- Mapa de distribución de Festuca patula Desf. en la Península Ibérica en cuadrículas UTM $10 \times 10 \mathrm{~km}$. Las citas bibliográficas contrastadas se indican en círculos negros, las nuevas localidades detectadas en el presente estudio en círculos blancos y las citas erróneas aparecen en círculos con borde en negrita.

Toledo (4) (Anexo I). En la mitad septentrional peninsular, la especie es mucho más rara y sólo existe una cita en el extremo occidental del Sistema Central, provincia de Salamanca (corroborada con pliego de herbario, HSS 299). Sin embargo, las citas de Festuca patula para el Caurel (Merino 1907) recogidas en la base de datos Anthos (http://www.anthos.es) han sido descartadas pues fueron redeterminadas como F. sylvatica (Litardiere 1956) y hoy se atribuyen a F. altissima (Fco. Javier Silva Pando (Herbario LOU) com. per.). Así mismo, la cita de Mayor (1975) en la cuenca del río Sorbe (Guadalajara) también fue descartada en la primera síntesis corológica para la especie (Cebolla \& Ponce 1990).

Características ambientales, ecológicas y demográficas de Festuca patula en Las Villuercas y Los Montes de Toledo. El estudio aportó información geoambiental, demográfica y ecológica de 20 poblaciones de $F$. patula de Las Villuercas y Los Montes de Toledo. Estas poblaciones se desarrollaban entre 300 y $1.000 \mathrm{~m}$ de altitud, ocupando litosuelos o suelos parcialmente desarrollados cuya roca madre era mayoritariamente de naturaleza metamórfica (cuarcitas, esquistos y pizarras). Se localizaban en áreas con temperaturas medias anuales suaves $\left(14-18^{\circ} \mathrm{C}\right)$ y unas precipitaciones medias anuales que oscilaban entre los 500 y $1.100 \mathrm{~mm}$ (Tabla 2). Tanto en Las Villuercas como en Los Montes de Toledo F. patula crecía en diferentes bosques de frondosas (Tabla 3), siendo común en las inmediaciones de arroyos o de pequeños ríos, en contacto con comunidades edafohigrófilas fre- 
Tabla 1

Nuevas poblaciones de Festuca patula de los Montes de Toledo, Las Villuercas y Sierra Nevada (CW, C y S de España) estudiadas en este trabajo. $N$ : tamaño poblacional. Cc: Cáceres, T: Toledo, CR: Ciudad Real, Gr: Granada, LV: Las Villuercas, M: Monfragüe, MT: Montes de Toledo y SN: Sierra Nevada. Especímenes de herbario de ocho de ellas han sido depositados en MA

\begin{tabular}{|c|c|c|c|c|c|c|}
\hline Población & Provincia & Municipio & Territorio & UTM & $\begin{array}{l}\text { Altitud } \\
(\mathrm{m})\end{array}$ & $N$ \\
\hline $\begin{array}{l}\text { Garganta de La Trucha } \\
\text { (MA 719533) }\end{array}$ & $\mathrm{Cc}$ & Alía & LV & 30SUJ0780 & 600 & 25 \\
\hline Río Viejas curso bajo & $\mathrm{Cc}$ & Cabañas del Castillo & LV & 30STJ8984 & 600 & 10 \\
\hline Río Viejas curso medio & $\mathrm{Cc}$ & Cabañas del Castillo & LV & 30STJ9083 & 640 & 150 \\
\hline $\begin{array}{l}\text { Garganta de Santa Lucía } \\
\text { (MA 719531) }\end{array}$ & $\mathrm{Cc}$ & Cabañas del Castillo & LV & $\begin{array}{l}\text { 30STJ8481; 30STJ8580; } \\
\text { 30STJ8581; 30STJ8579 }\end{array}$ & $520-540$ & 1.500 \\
\hline Río Ruecas (MA 719532) & $\mathrm{Cc}$ & Cañamero & LV & 30STJ9368 & 650 & 50 \\
\hline Garganta de Calabazas & $\mathrm{Cc}$ & Castañar de Ibor & LV & $\begin{array}{c}\text { 30STJ9689; 30STJ9589; } \\
30 \text { STJ9690 }\end{array}$ & $600-760$ & 61 \\
\hline $\begin{array}{l}\text { Arroyo del Chorreón } \\
\text { (MA719535) }\end{array}$ & $\mathrm{Cc}$ & Garvín & LV & 30STJ9497 & 500 & 50 \\
\hline Arroyo del Avellano & $\mathrm{Cc}$ & Navalvillar de Ibor & LV & 30STJ9186 & $530-550$ & 1.000 \\
\hline $\begin{array}{l}\text { Los Chapatales } \\
\text { (MA719536) }\end{array}$ & $\mathrm{Cc}$ & Navalvillar de Ibor & LV & 30STJ9381 & $600-650$ & 311 \\
\hline Garganta Salóbriga & $\mathrm{Cc}$ & Navalvillar de Ibor & LV & 30STJ9485 & 700 & 120 \\
\hline Charco Oscuro & $\mathrm{Cc}$ & Villar del Pedroso & LV & 30SUJ0086 & 680 & 52 \\
\hline La Pedriza (MA 719530) & $\mathrm{Cc}$ & Villar del Pedroso & LV & 30SUJ0084; 30SUJ0085 & $800-820$ & 200 \\
\hline Las Pasaderas & $\mathrm{Cc}$ & Villar del Pedroso & LV & 30SUJ0282 & $850-860$ & 15 \\
\hline Hoya de Guadarranque & $\mathrm{Cc}$ & Villar del Pedroso & LV & 30SUJ0483 & $660-680$ & 16 \\
\hline Castillo de Monfragüe & $\mathrm{Cc}$ & Torrejón el Rubio & M - LV & 29SQE5213 & 340 & 20 \\
\hline $\begin{array}{l}\text { Arroyo del Robledillo } \\
\text { (MA 719534) }\end{array}$ & CR & Navas de Estena & MT & 30SUJ6865; 30SUJ6965 & $600-700$ & 100 \\
\hline Arroyo Bénejar & Gr & Aldeire & SN & 30SVG9407 & 1.500 & 75 \\
\hline Río Fresnedoso & To & Espinoso del Rey & MT & 30SUJ4686 & 800 & 98 \\
\hline Río Frío & To & Los Navalucillos & MT & 30SUJ5970 & 700 & 5 \\
\hline Las Lanchas (MA719539) & To & Robledo del Mazo & MT & 30SUJ3782 & 960 & 302 \\
\hline Río Gévalo & To & Robledo del Mazo & MT & 30SUJ3890 & 600 & 6 \\
\hline
\end{tabular}

cuentes en riberas de arroyos y manantiales. La mayoría de las poblaciones de Las Villuercas y Los Montes de Toledo estaban integradas por menos de 100 ejemplares y solamente un par de poblaciones alcanzaban o superaban los 1.000 individuos. El tamaño poblacional dependía sig- nificativamente del tipo de suelo $(F=11,858, p=0,018)$, y era independiente de la altitud $(F=0,357, p=0,559)$. Las poblaciones más grandes se desarrollaban sobre suelos profundos, poco rocosos y, generalmente, solían estar en ambientes umbrosos, aunque la variable "insola- 
Tabla 2

Características geoambientales de las localidades con presencia de Festuca patula en la Península Ibérica.

Las provincias se indican de manera abreviada según Flora Ibérica

\begin{tabular}{|c|c|c|c|c|c|}
\hline $\begin{array}{l}\text { Territorio } \\
\text { (provincias) }\end{array}$ & $\begin{array}{l}\text { Rango } \\
\text { altitudinal } \\
(\mathrm{m})\end{array}$ & Litología & Suelo & $\begin{array}{c}\mathrm{P} \\
(\mathrm{mm})\end{array}$ & $\begin{array}{l}\mathrm{T}^{\mathrm{a}} \\
\left({ }^{\circ} \mathrm{C}\right)\end{array}$ \\
\hline $\begin{array}{l}\text { Las Villuercas y P.N. } \\
\text { Monfragüe (Cc) }\end{array}$ & $340-860$ & $\begin{array}{l}\text { cuarcitas, pizarras, } \\
\text { esquistos }\end{array}$ & $\begin{array}{l}\text { suelos rocosos -AC, ARC o } \\
\text { (A)RC- y parcialmente } \\
\text { desarrollados -A(B)C- }\end{array}$ & $600-1.100$ & $14,3-17,7$ \\
\hline Montes de Toledo (CR, To) & $500-900$ & $\begin{array}{l}\text { cuarcitas, pizarras, } \\
\text { esquistos }\end{array}$ & $\begin{array}{l}\text { suelos rocosos -AC, ARC o } \\
\text { (A)RC- y parcialmente } \\
\text { desarrollados -A(B)C- }\end{array}$ & $500-800$ & $14,4-15,2$ \\
\hline Sierra Morena (CR, Co, J, Se) & $300-1.050$ & $\begin{array}{l}\text { cuarcitas, pizarras, } \\
\text { esquistos }\end{array}$ & $\begin{array}{l}\text { suelos rocosos o parcialmente } \\
\text { desarrollados }\end{array}$ & $700-900$ & $14,6-15,6$ \\
\hline $\begin{array}{l}\text { Cordilleras Béticas } \\
(\mathrm{A}, \mathrm{Al}, \mathrm{Ca}, \mathrm{Co}, \mathrm{Gr}, \mathrm{J}, \mathrm{Ma})\end{array}$ & $640-1.750$ & $\begin{array}{l}\text { rocas carbonatadas, } \\
\text { esquistos }\end{array}$ & suelos rocosos & $450-1.950$ & $11,7-16,4$ \\
\hline Sistema Central (Sa) & $500-700$ & cuarcitas & - & 1.100 & $13,9-14,7$ \\
\hline
\end{tabular}

ción" no resultó estadísticamente significativa $(F=0,067$, $p=0,798)$.

\section{DISCUSIÓN}

Distribución ibérica de Festuca patula. De la primera síntesis corológica realizada de para la Península Ibérica (Cebolla \& Ponce 1990) se desprendía que F. patula era una planta propia del tercio sur peninsular pues la mayoría de las localidades se encontraban en las Cordilleras Béticas. Posteriormente, se incrementó el conocimiento corológico haciéndose más patente la presencia de la especie el borde norte de Sierra Morena (García 1999, 2004, 2006). Sin embargo, la existencia de F. patula estaba es- casamente documentada en el centro oeste de la Península Ibérica. Sólo se había citado en algunas localidades en las estribaciones meridionales de Los Montes de Toledo (Cebolla \& Ponce 1990, 1993a, 1993b) y en Extremadura se describía como una gramínea rara (Devesa 1995) para que únicamente había pliegos de tres localidades dispersas (Anexo I: HSS 4583, Badajoz, La Codosera, río Gevorete; HSS 2633 y HSS 2635, Cáceres, Navalvillar de Ibor; MA 522247 y MA 522249, Cáceres, Monfragüe).

Sin embargo, las prospecciones de campo realizadas proporcionan un considerable número de localidades nuevas con las que se incrementa notablemente el área de distribución conocida de la especie en la península, especialmente en Los Montes de Toledo y en las sierras extremeñas de Las Villuercas. No obstante, pese la notable ampliación

Tabla 3

Comunidades vegetales en las que crecen las poblaciones ibéricas estudiadas de Festuca patula. Entre paréntesis número de poblaciones instaladas en suelos muy rocosos (litosuelos). Beh: bosque edafo-higrófilo

\begin{tabular}{lcccccccccc}
\hline \multicolumn{1}{c}{ Territorio } & Pinsapar & $\begin{array}{c}\text { Melojar- Alcornocal- } \\
\text { Castañar }\end{array}$ & Quejigar & Encinar & $\begin{array}{c}\text { Encinar- } \\
\text { Beh }\end{array}$ & Beh & $\begin{array}{c}\text { Matorral } \\
\text { Batorral- } \\
\text { Beh }\end{array}$ & Herbazal Roquedo \\
\hline $\begin{array}{l}\text { Las Villuercas-P. N. } \\
\text { Monfragüe }\end{array}$ & $2(1)$ & & $6(2)$ & $3(2)$ & & & $6(5)$ & & & \\
Montes de Toledo & & 1 & 1 & 2 & $1(1)$ & $1(1)$ & & $2(2)$ & & \\
$\begin{array}{l}\text { Sierra Morena } \\
\text { Cordilleras Béticas }\end{array}$ & 2 & $1(1)$ & 3 & 1 & $1(1)$ & $5(1)$ & 1 & & & $2(2)$ \\
Sistema Central & & 1 & & 2 & $2(1)$ & & 8 & 1 & $1(1)$ & $7(7)$ \\
\hline
\end{tabular}


de distribución, la existencia (corroborada con pliego herbario, Anexo I: HSS 299) en la vertiente norte del Sistema Central occidental y en el extremo noroccidental de Badajoz, sugiere que su área puede ser aún mayor en el centro y oeste de la Península Ibérica.

Ecología de Festuca patula. Nuestros análisis han demostrado que F. patula muestra una importante amplitud ecológica en el CW de la Península Ibérica. La especie ocupa un rango altitudinal considerable (c. $700 \mathrm{~m}$, rango entre 300-1.000 m) en áreas con un clima mediterráneo templado, moderadamente cálido $\left(14-18^{\circ} \mathrm{C}\right.$, precipitación 500-1.100 mm). En Sierra Morena y en el Sistema Central se localiza en cotas altitudinales similares y en regiones con un clima muy parecido. Sin embargo, en las Cordilleras Béticas suele encontrarse por encima de los 700 $m$ de altitud $y$, en enclaves de Sierra Nevada o en las sierras de Pandera, alcanza y supera los $1.500 \mathrm{~m}$. En las Cordilleras Béticas las poblaciones de F. patula se sitúan en áreas con notables diferencias termopluviométricas con respecto a Las Villuercas y Los Montes de Toledo (Tabla 2) pues hay localidades con precipitaciones mucho más elevadas (por ejemplo en Grazalema $>1.900 \mathrm{~mm}$ ) y con temperaturas medias más bajas (p. ej. Cazorla $11,7^{\circ} \mathrm{C}$ ) (Anexo II).

Desde el punto de vista geológico, las poblaciones del CW ibérico se diferencian de las de otras regiones peninsulares por su exclusiva localización sobre sustratos de naturaleza ácida (cuarcitas, esquistos y pizarras); en concordancia con la litología silicícola predominante en Las Villuercas y en Los Montes de Toledo donde las rocas carbonatadas son muy escasas (Gibbons \& Moreno 2002). En el resto de la península F. patula no sólo coloniza ambientes silíceos sino que también aparece sobre sustratos carbonatados por lo que ha de considerarse una especie indiferente edáfica. En efecto, en Sierra Morena, en Sierra Nevada y en el Sistema Central se desarrolla sobre suelos y litosuelos de cuarcitas u otras rocas metamórficas paleozoicos. Pero en las Cordilleras Béticas, desde Cádiz hasta Alicante, la mayoría de las poblaciones crecen en suelos o litosuelos de naturaleza básica, sobre rocas calizas y dolomías jurásicas y cretácicas (http://www.igme.es).

Los análisis estadísticos de regresión lineal confirman que las poblaciones del $\mathrm{CW}$ ibérico que crecen en suelos profundos, son significativamente mayores que las que crecen en litosuelos. No obstante, su presencia en estos últimos (incluyendo fisuras de paredes escarpadas) indican que podría considerarse como una especie rupícola facultativa como también se desprende de las descripciones recopiladas para las Cordilleras Béticas (Rivas Goday \&
Rivas Martínez 1969, Cebolla \& Ponce 1990, Llamas et al. 2002) y para Sierra Morena (Cebolla \& Ponce 1990).

La amplitud ecológica de F. patula se manifiesta igualmente en la variedad de comunidades vegetales en las que se ha comprobado que vive, tanto en el CW de España como en otras zonas peninsulares (Tabla 3). Así, no sólo habita en bosques o matorrales esclerófilos mediterráneos (Rivas Goday \& Rivas Martínez 1969, Cebolla \& Ponce 1990, García 2006, Catalán 2009) sino también en fondos de barrancos 0 en las inmediaciones de cauces hídricos, formando parte de la orla o el cortejo florístico de las comunidades riparias 0 edafohigrófilas (Cebolla \& Ponce 1990; García 1999, Calleja \& Sainz 2009). En el resto de las regiones peninsulares la planta ha sido citada en ambientes similares, además de en pinsapares (Aparicio \& Silvestre 1987), en roquedos (Cebolla \& Ponce 1990) o en herbazales (Serra et al. 2000) (Tabla 3). Habida cuenta de la versatilidad ambiental de F. patula y la abundancia de las comunidades vegetales en las que habita, sería esperable que la planta fuese más frecuente y presentase poblaciones más grandes. Sin embargo, no es una planta común y la mayoría de sus poblaciones $\mathrm{CW}$ ibéricas constan de pocos individuos. Las causas de la rareza y de las bajas densidades poblacionales de la especie podrían deberse, entre otros factores, a descensos demográficos experimentados por sus poblaciones durante las fases glaciales Pleistocénicas, dada la relativa antigüedad de esta planta (Inda et al. 2008), 0 a la incidencia humana. La presión histórica y actual de la elevada densidad de herbívoros domésticos (Mateos-Quesada 2007) y cinegéticos (Charco 2002, Pulido et al. 2007) son factores que podrían explicar también su actual escasez en los hábitats relativamente más comunes y a su vez propicios para la especie -sotobosques con suelos desarrollados- por ser accesibles a los herbívoros.

\section{AGRADECIMIENTOS}

Este trabajo se inició gracias a la formación y apoyo recibido por parte de Jose Ángel López-Rodríguez. Por otra parte, agradezco el permiso de Mauricio Velayos y la diligente ayuda de Charo Noya Santos en la consulta de pliegos de Festuca patula depositados en Real Jardín Botánico de Madrid. Así mismo, quisiera destacar la rápida respuesta y la información prestada por Salvador Talavera sobre los pliegos de Festuca patula disponibles en el herbario de Sevilla; igualmente agradezco la disponibilidad de Francisco Javier Silva Pando (Herbario de Lourizán) y de Consuelo Cebolla (Dpto. Biología, UAM) para resolver dudas sobre las citas y pliegos de Festuca recogidos por Pere Merino en Galicia y por M. Mayor en el este del Sistema Central. El manuscrito experimentó una notable mejora gracias a las correcciones y sugerencias de un revisor anónimo. 


\section{BIBLIOGRAFÍA}

Aparicio, A. \& Silvestre, S. 1987. Flora del Parque Natural de la Sierra de Grazalema. Junta de Andalucía, Agencia de Medio Ambiente, Sevilla.

Cabezudo, B.; Pérez Latorre, A. V.; Navas Fernández, D.; Gavira, Ó. \& Caballero, G. 2005. Contribución al conocimiento de la flora del Parque Natural de las Sierras Tejeda, Almijara y Alhama (Málaga-Granada, España). Acta Bot. Malac. 30: 55-110.

Calleja, J. A. \& SAinz, H. 2009. Análisis e interpretación geobotánica de la estructura y composición florística de las loreras ibéricas. Ecología 22: 45-71.

Catalán, P. 2009. Festuca L. En: G. Blanca, B. Cabezudo, M. Cueto, C. Fernández López \& C. Morales (Eds.), Flora Vascular de Andalucía Oriental 1: 298-310. Consejería de Medio Ambiente, Junta de Andalucia, Sevilla.

Cebolla, C. \& Rivas-Ponce, M. A. 1990. Mapa 471. Festuca triflora Desf. En: J. F. Casas \& R. Gamarra (Eds.), Asientos para un atlas corológico de la flora occidental, 17, Fontqueria 30: 213.

Cebolla, C. \& Rivas-Ponce, M. A. 1993a. Adiciones. Mapa 471. Fontqueria 36: 206.

Cebolla, C. \& Rivas-Ponce, M. A. 1993b. Las gramíneas de Ciudad Real. Fontqueria 36: 306-347.

Costa, M.; Morla, C. \& Sainz, H. (Eds.). 1997. Los bosques ibéricos. Planeta, Barcelona.

Charco, J. 2002. Una introducción al estudio de la velocidad de regeneración natural del bosque mediterráneo y de los factores antropozoógenos que la condicionan. En: J. Charco (Ed.), La regeneración natural del bosque mediterráneo en la Península Ibérica. Evaluación de problemas y propuesta de soluciones: 115-149. ARBA-MIMAM, Madrid.

DevesA, J. A. 1991. Las gramíneas de Extremadura. Servicio de Publicaciones de la Universidad de Extremadura, Badajoz.

Devesa, J. A. 1995. Vegetación y flora de Extremadura. Universitas Editorial, Badajoz.

De la Torre, A.; Alcaraz, F. \& Serra, L. 1996. Aportaciones a la flora alicantina (SE de España), II. Anales Biol., Fac. Biol., Univ. Murcia 21: 73-80.

Fernández Liencres, J. \& Fernández López, C. 1996. Flórula del río Cañamares (Jaén). Blancoana 13: 69-80.

FernÁndez López, C. \& Gómez Montabés, V. 1995. Plantas de la Pandera en el Herbario Jaén hasta 1993. Blancoana 12: 1-7.

FonT, L. 1983. Atlas climático de España. Instituto Nacional de Meteorología, Madrid.

GARCíA, R. 1999. Aportaciones a la flora de Sierra Morena (Ciudad Real, España). Bot. Complut. 23: 115-132.

García, R. 2004. Flora vascular de Sierra Madrona y su entorno (Sierra Morena, Ciudad Real, España). Ecología 18: 147-214.

GARCíA, R. 2006. Flora y vegetación de Sierra Madrona y Valle de Alcudia. Centro de Investigaciones Ambientales del Mediterráneo, Ciudad Real.
Gibbons, W. \& Moreno, T. 2002. Geology of Spain. The Geological Society Publishing House, London.

Herrero, J.; Cirujano Bracamonte, S.; Moreno Pérez, M.; Peris Gisbert, J. B. \& Stübing Martínez, G. 2003. La vegetación protegida en Castilla La Mancha. Junta de Comunidades de Castilla-La Mancha, Toledo.

Inda, L. A.; Segarra-Moragues, J. G.; Müller, J.; Peterson, P. M. \& Catalán, P. 2008. Dated historical biogeography of the temperate Loliinae (Poaceae, Pooideae) grasses in the northern and southern hemispheres. Molec. Phylogen. Evol. 46 (3): 932-957.

LAdero, M. 1987. España Lusoextremadurense. En: M. Peinado \& S. Rivas Martínez (Eds.), La vegetación de España: 453-486. Universidad de Alcalá de Henares, Alcalá de Henares.

Litardiere, R. 1956. Contribution a l'etude des Festuca de Galice d'apres les recoltes du Pere Merino. Bol. Soc. Brot. Ser. C. N. 24(1): 1-18.

Llamas, F.; Acedo, C.; Penas, A. \& Pérez Morales, C. 2002. Una nueva subespecie de Festuca paniculata en la Cordillera Cantábrica. Lagascalia 22: 119-129.

Mateos-Quesada, P. 2007. Antaño en Las Villuercas. Una mirada retrospectiva hacia el interior de nuestra cultura. Asociación por la Arquitectura Rural Tradicional de Extremadura. Cáceres.

Mayor, M. 1975. Datos florísticos sobre la Cordillera Central (Somosierra, Ayllón y Pela). Anales Inst. Bot. Cavanilles 32: 323-347.

Merino, B. 1907. Viajes de herborización por Galicia. Área de Ciencias Agrarias, Seminario de Estudios Galegos, La Coruña.

Molero Mesa, J. \& PÉrez-Raya, F. 1987. La flora de Sierra Nevada. Universidad de Granada. Excma. Dip. Prov. Granada.

Mueller, J. \& CATAlán, P. 2006. Notes on the infrageneric classification of Festuca L. (Gramineae). Taxon 55: 139-144.

MuÑoz, J. \& SAnz, C. 1995. Las Montañas, Guía física de España. Alianza Editorial. Madrid.

Pulido, F.; SAnz, R.; ABel, D.; EzQuerra, J.; GIL, A.; GonzÁLez, G.; Hernández, A.; Moreno, G.; Pérez, J. J. \& VÁZQUEZ, F. M. 2007. Los bosques de Extremadura. Evolución, ecología y conservación. Consejería de Industria, Energía y Medio Ambiente. Junta de Extremadura, Mérida.

Rivas Goday, S. \& Rivas Martínez, S. 1969. Matorrales y tomillares de la Península Ibérica comprendidos en la clase Ononido-Rosmarinetea Br.-B1. 1947. Anales Inst. Bot. Cavanilles 25: 5-197.

Serra, L.; Fabregat, C.; Herrero-Borgoñón, J. J. \& López, S. 2000. Distribución de la flora vascular endémica, rara o amenazada en la Comunidad Valenciana. Generalitat Valenciana, Valencia.

Valdés, B.; Redjali, M.; Achhal, A.; Jury, S. L. \& MontseRRAT, J. M. (Eds.) 2002. Catalogue des plantes vasculaires du Nord de Maroc. CSIC, Madrid. 
Anexo I

Localidades con coordenadas de Festuca patula en la Península Ibérica ordenadas por provincias. Para cada localidad se indica: i: el territorio en el que se encuentra, $\mathrm{CB}=$ Cordilleras Béticas, LV-M = Las Villuercas-Monfragüe, MT= Montes de Toledo, $\mathrm{SC}=$ Sistema Central, SM=Sierra Morena; ii: la coordenada en cuadrículas UTM $1 \times 1 \mathrm{~km} 010 \times 10 \mathrm{~km}$; iii: fuente de la cita (bibliográfica, pliego de herbario), BC=Herbario Instituto Botánico de Barcelona; $\mathrm{ABH}=$ Herbario de la Universidad de Alicante, HSS = Herbario La Orden-Valdesequera-Junta de Extremadura, HUAL = Herbario de la Universidad de Almería, JACA = Herbario de Jaca, LEB = Herbario de la Universidad de León, MA= Herbario Real Jardín Botánico de Madrid, MAF= Herbario de la Facultad de Farmacia de Madrid, MUB= Herbario de la Universidad de Murcia, REDIAM-

CMA = Junta de Andalucía, SEV = Herbario de la Universidad de Sevilla; iii) localidad precisa y altitud (contenida en el la referencia bibliográfica, en el pliego de herbario o la obtenida en la prospección de campo); iv) fecha de recolección; v) colector o responsable de la cita.

ESPAÑA: ALICANTE: (CB) 30SYH0664, De la Torre et al. 1996, MUB 31659, Tibi, Sierra del Maigmó, carrascales, $1.080 \mathrm{~m}, 21-\mathrm{VI}-1990$, De la Torre; (CB) 30SYH0664, Serra 2007, ABH 6647, Tibi, Serra del Maigmó, presente en pastizales sobre suelos pedregosos umbríos dominados por gramíneas y en contacto con bosques mixtos y encinares, 1.080 m, 21-VI-1989, De la Torre; (CB), 30SYH0664, Serra 2007, Tibi, Serra del Maigmó, pr. Coll de la Xau, presente en pastizales sobre suelos pedregosos umbríos dominados por gramíneas y en contacto con bosques mixtos y encinares, $1.100 \mathrm{~m}$, 6-VII-2004, Serra \& Pérez; (CB), 30SYH1482, ABH-6800, Alcoi, Serra del Carrascal d'Alcoi, presente en pastizales sobre suelos pedregosos umbríos dominados por gramíneas y en contacto con bosques mixtos y encinares, $1.100 \mathrm{~m}, 13-\mathrm{VI}-1993$, Cristóbal \& Isidro; (CB), 30SYH1482, Serra et al. 2000, Alcoi, Serra del Carrascal d'Alcoi, 1.100 m. ALBACETE: (CB), 30SWH45, Cebolla \& Rivas Ponce 1993a, Riopar, nacimiento del río Mundo, WH4956, 1.200 m, 9VI-1992, Cebolla \& Rivas Ponce. Almería: (CB), 30SWF1288, HUAL 5636-1, Sa de Gádor, Morrón de Los Colorados, VI-1992; (CB), 30SWG77, Cebolla \& Rivas Ponce 1990, BC 69542, Peñas del Barrancón, c. María, 1.700 m, 26-VI-1921, Gros. (CB), 30SWG77, Cebolla \& Rivas Ponce 1990, MA 12424, Peñas del Barrancón, c. María, 1.700 m, 26-VI-1921, Gros. BaDAJ0Z: 29SPD5444, HSS 4583, La Codosera, márgenes del río Gevorete, alisedas y proximidades de alcornocal, 1998-1011, Gadea \& Vázquez. CÁdIZ: (CB), 30STF8372, SEV 205030, Grazalema, Sierra del Pinar, El Pinsapar, TF8577, 1.200 m, 28/06/1984, Aparicio et al. (CB), 30STF8571, Aparicio \& Silvestre 1987, Grazalema, Sierra del Pinar, El Pinsapar, 900 m; (CB), 30STF8574, Aparicio \& Silvestre 1987, Grazalema, Cerro del Montón, arroyo Los Ballesteros, 900 m; (CB), 30STF8577, SEV 205030, Grazalema. Sierra del Pinar. El Pinsapar, TF8577, 1.200 m, 28/06/1984, Aparicio et al.; (CB), 30STF86, Cebolla \& Rivas Ponce 1990, BC-111125, Benaocaz, 24-VI-25, Gros; (CB), 30STF86, Cebolla \& Rivas Ponce 1990, BC-111112, Benaocaz, Caillo, 1.200, 24-VI-25, Font Quer \& Gros; (CB), 30STF86, BC-111125, Benaocaz, 1.200 m, 24-VI-25, Gros; (CB), 30STF86, BC-111112, Benaocaz, Caillo, $1.200 \mathrm{~m}, 24-\mathrm{VI}-25$, Font Quer \& Gros; (CB), 30STF87, SEV 97372, Grazalema, cercanías, 6-V-81, García Rowe \& Silvestre; (CB), 30STF87, Cebolla \& Rivas Ponce 1990, SEV 112565, Grazalema, cerro el Montón, 14-V-1984, Aparicio et al.; (CB), 30STF87, Cebolla \& Rivas Ponce 1990, BC-111110, Grazalema, Sierra del Pinar, 12-VII-1925, Gros; CÁCERES: (LV-M), 29SQE5213, Torrejón el Rubio, umbría del Castillo de Monfragüe, 340 m, Calleja; (LV-M), 30STJ8481, MA 719531, Cabañas del Castillo, Garganta de Santa Lucía, 30STJ8481, 540 m, sotobosque de encinas. Calleja; (LV-M), 30STJ8579, Cabañas del Castillo, Garganta de Santa Lucía, 540 m, Calleja; (LV-M), 30STJ8580, Cabañas del Castillo, Garganta de Santa Lucía, 530 m, Calleja; (LV-M), 30STJ8581, Cabañas del Castillo, Garganta de Santa Lucía, 525 m, Calleja; (LV-M), 30STJ8984, Cabañas del Castillo, Río Viejas, 600 m, Calleja; (LV-M), 30STJ9083, Cabañas del Castillo, Río Viejas, 640 m, Calleja; (LV-M), 30STJ9186, Navalvillar de Ibor, Arroyo del Avellano, 530-550 m, Calleja; (LV-M), 30STJ9368, MA 719532, Cañamero, río Ruecas, 30STJ9368, $650 \mathrm{~m}$, orilla rocosa, Calleja; (LV-M), 30STJ9381, MA 719536, Naval- villar de Ibor, Los Chapatales, 30STJ9381, 600-650 m, sotobosque encinar arborescente, Calleja; (LV-M), 30STJ9485, Navalvillar de Ibor, Garganta Salóbriga, 700 m, Calleja; (LV-M), 30STJ9497, MA 719535, Garvín, arroyo del Chorreón, río Gualija, 30STJ9497, 500 m, ladera rocosa con encinar arborescente junto a ribera, Calleja; (LV-M), 30STJ9589, Castañar de Ibor, Garganta de Calabazas, 820 m, Calleja; (LV-M), 30STJ9689, Castañar de Ibor, Garganta de Calabazas, 760 m, Calleja; (LV-M), 30STJ9690, Castañar de Ibor, Garganta de Calabazas, 600 m, Calleja; (LV-M), 30STJ98, HSS 2633, 2635, Navalvillar de Ibor, en bosques de rebollo, zonas de ribera y turberas, 1997-9-16, González et al.; (LV-M), 30STK40, MA 522247, Monfragüe, 400 m, TK40, 28/04/1988, Devesa \& Ortega; (LV-M), 30STK40, MA 522249, Monfragüe, inmediaciones del Castillo, 30-04-1987, Devesa \& Vázquez; (LV-M), 30SUJ0084, MA 719330, Villar del Pedroso, La Pedriza, 30SUJ0084, 800-820 m, márgenes de ribera, laderas pedregosas bajo castaños y robles, Calleja; (LVM), 30SUJ0085, Villar del Pedroso, La Pedriza, 760-780 m, Calleja; (LVM), 30SUJ0086, Villar del Pedroso, Garganta del Mesto, Charco Oscuro, $680 \mathrm{~m}$, Calleja; (LV-M), 30SUJ0282, Villar del Pedroso, Las Pasaderas, 850-860 m, Calleja; (LV-M), 30SUJ0483, Villar del Pedroso, Hoya de Guadarranque, 660-680 m, Calleja; (LV-M), 30SUJ0780, MA 719533, Alía, La Trucha, 30SUJ0780, 600 m, pared rocosa, entre Juniperus oxycedrus, Fraxinus anfustifolia, Arbutus, mucho Polypodium vulgare y vegetación riparia con Alnus, Calleja. CóRdoba: (CB), 30SUG83, Cebolla \& Rivas Ponce 1990, SEV-6317, Priego de Córdoba, Sierra Horconera, VI-1959, Borja; (CB) Sierra Horconera, umbría de las grietas de los roquedos y en sotobosque del encinar quejigal, VI-1959, Borja; (CB), 30SUG83, Cebolla \& Rivas Ponce 1990, MAF-70089, MAF-72161, MAF-103438, MA-200642, Borja; (SM), 30STG7065, REDIAM-CMA 188709, Hornachuelos, 300 m; (SM), 30STG9690, REDIAM-CMA 188708, Hornachuelos, 200 m; (CB), 30SUG85, Unex 01358-1, Zuheros, entre Lobatejo y Ermita de la Virgen de la Sierra, roquedos, calizas, encinar, 1.000 m, 1984-7-3, Devesa \& Pastor; (SM), 30SUH10, Cebolla \& Rivas Ponce 1990, SEV-97715, Posadas, hacia Villaviciosa, arroyo Pajaroncillo, 18-V-80, Corral \& Fernández Corrales; (SM), 30SUH37, Cebolla \& Rivas Ponce 1990, SEV 103628, Santa Eufemia, 800 m, 27-V82, Devesa \& García Rowe. Ciudad ReaL: (SM), 30SUH8551, Cebolla \& Rivas Ponce 1993b, Fuencaliente, 1k de Fuencaliente, 700 m; (SM), 30SUH8551, Cebolla \& Rivas Ponce 1990, JACA 40863, Fuencaliente, alcornocal con Quercus canariensis, sobre suelos con esquistos-cuarcitas, umbría fresca, barranco junto al río en el desfiladero, a $1 \mathrm{~km}$ de Fuencaliente, 700 m, 7-VI-1963, Montserrat \& Sandwith; (SM), 30SUH8551, García 1999, Fuencaliente, río Jerrumbrosa, 760 m; (SM), 30SUH8551, MA-597189, Fuencaliente, Jerrumbrosa, 760 m, 30SUH8551, 19-V-1996; (SM), 30SUH8554, García 1999, Fuencaliente, valle del río Pradillo, 740 m; (SM), 30SUH8554, MA-597191, Fuencaliente, valle del río Pradillo, $740 \mathrm{~m}$, alcornocal-quejigos en las proximidades de arroyuelos, 30SUH8554, 23-05-1996, García Río \& Barrios Pérez; (SM), 30SUH8754, Fuencaliente, Arroyo Cereceda, 740 m, Calleja; (MT), 30SUJ56, Cebolla \& Rivas Ponce 1993b, río Estena; (MT), 30SUJ6423, Cebolla \& Rivas Ponce 1993a, Puebla de Don Rodrigo, río Guadiana, 
UJ6423, 500 m, Cebolla \& Rivas Ponce, 21-IV-1991; (MT), 30SUJ6521, Cebolla \& Rivas Ponce 1993a, Saceruela, Castilnegro, vert. NW, UJ6521, 820 m, Cebolla \& Rivas Ponce, 21-IV-1991; (MT), 30SUJ6522, Cebolla \& Rivas Ponce 1993a, Puebla de Don Rodrigo, Castilnegro, vert. N, 560 m, Cebolla \& Rivas Ponce, 21-IV-1991; (MT), 30SUJ67, Cebolla \& Rivas Ponce 1990, MAF 27315, En los robledales del río Estena, 13-V-1977, Velasco Negueruela; (MT), 30SUJ6865, MA 719534, Navas de Estena, arroyo Robledillo, 600-700 m, orillas rocosas entre madroñal y loros y fresnos, Calleja; (MT), 30SUJ6954, ABH 49397-1, Alcoba, Valle de los Enamorados, 2004-5-13, Cristóbal et al:; (MT), 30SUJ6965, Navas de Estena, Arroyo del Robledillo, 600-700 m, Calleja; (SM), 30SVH0153, García 1999, Solana del Pino, Sierra Madrona, Garganta del Almagrero, 1020 m; (SM), 30SVH0153, MA-597215, Solana del Pino, Sa Madrona, Garganta Almagrero 1020, 14-VII-1997, García Río; (SM), 30SVH0460, García 1999, Solana del Pino, Sierra Madrona, umbría de las Tiñosas, 900 m; (SM), 30SVH0460, MA-597192, Solana del Pino, Sa Madrona, umbría de las Tiñosas, 900 m, 3-V-1997, García Rio; (SM), 30SVH1549, García 1999, Mestanza, Hoz del Jándula, 420 m; (SM), 30SVH1549, MA 597190, Mestanza, Hoz río Jándula, 420 m, 20-VI-1996, García Río \& Barrios Pérez; (SM), 30SVH2460, MA 729513, San Lorenzo de Calatrava, Barranco de la Hocedilla, esquistos, cauce del arroyo, $690 \mathrm{~m}$, 18/V/2002. Carrasco et al. Granada: (CB), 30SUF89, Rivas Goday \& Rivas Martínez 1969, Puerto de Alazores, 950 m; (CB), 30SVF19, Rivas Goday \& Rivas Martinez 1969, Alhama de Granada, a Loja, 900 m; (CB), 30SVF0985, Cabezudo et al. 2005, Alhama de Granada; (CB), 30SVG70, Molero et al. 1987, Valle río Genil; (CB), 30SVG9407, Aldeire, Arroyo Benéjar, 1.500 m, Calleja; (CB), 30SWG2296, Cebolla \& Rivas Ponce 1993a, Castril, Sierra de Castril, barranco de las Palomas. JAÉN: (CB), 30SVG2478, MA 554243, SEV 233567, (CB), 30SVG3165, Fernández \& Montabes 1995, La Pandera, $1.750 \mathrm{~m}$; (CB), 30SVG3566, MA 409508, Pantano de Quiebrajano, arroyo de los ladrones, 700 m, 9/06/1976, Fernández Casas et al.; (CB), 30SVG57, Cebolla \& Rivas Ponce 1990, macizo de Mágina, Sierra Almadén, vertiente W, 1600-1700 m, encinar, 2V-1988, Cebolla; (CB), 30SVG67, Cebolla \& Rivas Ponce 1990, BC 69544, In declivibus Magina Orientalis, Las Pilas, in rupestribu calcareis, 1.700 m, 5-VII-1925, Cuatrecasas; (CB), 30SVG67, BC 69544, In declivibus Magina Orientalis, Las Pilas, in rupestribu calcareis, $1.700 \mathrm{~m}$, 5-VII-1925, Cuatrecasas; (SM), 30SVH54, Cebolla \& Rivas Ponce 1990, MAF-70088, MA-200641, MA-181356, Despeñaperros, umbrías, roquedos, VI-1963, Borja; (SM), 30SVH54, Cebolla \& Rivas Ponce 1990, MA170212, Despeñaperros, 6-VI-1963, Fernández Galiano; (SM), 30SVH54, Cebolla \& Rivas Ponce 1990, MAF-27315, Valdeazores, Despeñaperros, 10-V-1941, Quercetum ilicis monspessulanum; (SM), 30SVH55, Cebolla \& Rivas Ponce 1990, JACA-166267, Valdeazores, barranco fresco con castaños y robles, 1.070 m, 27-V-67, Montserrat; (CB), 30SVH9912, MA 714544-1, Villacarrillo, Mogon Casa de Fuente Higuera, 800 m, 1996-619, Rodríguez Torres \& Fdez. Ocaña; (CB), 30SWG0197, Cebolla \& Rivas Ponce 1990, BC-641791, La Iruela, Fuente de la Raja, 22-V-1981,
Hernández Cardona; (CB), 30SWG0197, Cebolla \& Rivas Ponce 1990, MA-246272, La Iruela, Fuente de la Raja, 22-V-1981, Hernández Cardona; (CB), 30SWG0197, Cebolla \& Rivas Ponce 1990, SEV 79343, La Iruela, Fuente de la Raja, 22-V-1981, Hernández Cardona; (CB), 30SWG0197, Cebolla \& Rivas Ponce 1990, El Valle, 17-IV-1974, Soriano; (CB), 30SWG0197, Cebolla \& Rivas Ponce 1990, Segura Cazorla, macizo, alrededores de la Fuente del Oso, grietas de lapiaz, 31-V-1976, Muñoz Garmendia \& Soriano; (CB), 30SWG0197, Cebolla \& Rivas Ponce 1990, Segura Cazorla, macizo, margen izquierda del arroyo de la Mesa, grietas de lapiaz, 28-V-1976, Muñoz Garmendia \& Soriano; (CB), 30SWG0197, Cebolla \& Rivas Ponce 1990, Segura Cazorla, macizo, sobre la cerrada del Utrero, grietas de lapiaz, 28-V-1976, Muñoz Garmendia \& Soriano; (CB), 30SWG0197, Llamas et al. 2002, LEB-16129, La Iruela, Fuente La Raja, ad rupes calcareas, 22-V-1981, Hernández; (CB), 30SWG0197, MA 362218, La Iruela, Fuente la Raja, 22-05-1981; (CB), 30SWG08, Cebolla \& Rivas Ponce 1990, Segura Cazorla, Cañada de las Fuentes, grietas de lapiaz, 29-V-1976, Muñoz Garmendia \& Soriano; (CB), 30SWH00, Cebolla \& Rivas Ponce 1990, Villacarrillo, Sierra de las Villas, Chorro Gil, 1.200 m, bujedo, 2-V-1987, Soriano; (CB), 30SWH01, Cebolla \& Rivas Ponce 1990, Villacarrillo, Sierra de las Villas, Aguascebas de Gil Cobo, 1.400 m, bujedo denso, 2-V-1987, Soriano; (CB), 30SWH0206, Fernández \& Fernández 1996, La Iruela, Cerrada de Cañamares, 700 m; (CB), 30SWH10, Cebolla \& Rivas Ponce 1990, MA 263325, BC 644241, Sierra de Cazorla, río Borosa, piscifactoria, 30V-1982, Hernández Cardona; (CB), (CB), 30SWH12, Cebolla \& Rivas Ponce 1990, Villanueva del Arzobispo, Sierra de las Villas, barranco de María, vertiente W, maleza, 640 M, 1-V-1987, Soriano; (CB), 30SWH32, SEV 204968, Poyotello, Sierra de Segura, gorge of river Segura, rocky and stony ground, calcareous soils, 1.425m, 26/06/1988, Alziar, Galland, Romero, Talavera, Valdés et al. MálaGa: (CB), 30STF97, Cebolla \& Rivas Ponce 1990, SEV 110126, Ronda, hacia Montecorto, 11-V-1984, Devesa et al.; (CB), 30SUF06, Cebolla \& Rivas Ponce 1990, BC 11109, Sierra de Ronda, 20-VII-1925, Gros; (CB), 30SUF26, Cebolla \& Rivas Ponce 1990, MA 12423, Sierra de las Nieves, bois du sapin, sur calcaire, rare, 12-VII-1889, Reverchon; (CB), 30SUF79, Rivas Goday \& Rivas Martinez 1969, Puerto de las Pedrizas, cerca, 850 m; (CB), 30SUF89, Rivas Goday \& Rivas Martinez 1969, Alfarnate, Sierra de Alazores, 1.000 m; (CB), 30SUG70, Rivas Goday \& Rivas Martinez 1969, Llanos de Archidona, a Loja, 700-750 m. Salamanca: (SC), 29TQE5284, HSS 299, Peña de Francia, Sequeros, bosques de rebollos y castaños, herbazales de sotobosque, 1996-6-29, Vázquez. SeviLLa: (SM), 30STH8797, REDIAMCMA 145094, Las Navas de la Concepción, 300 m, 1993-6-24. ToLEDo: (MT), 30SUJ3782, MA 719539, Las Hunfrías, Robledo del Mazo, Gargantas de las Lanchas, $960 \mathrm{~m}$, ladera rocosa, lorera con abedules, acebos, tejos y arraclanes, Calleja; (MT), 30SUJ3890, Robledo del Mazo, río Gévalo, $600 \mathrm{~m}$, Calleja; (MT), 30SUJ4686, Espinoso del Rey, río Fresnedoso, 800 m, Calleja; (MT), 30SUJ5970, Los Navalucillos, río Frío, 700 $\mathrm{m}$, Calleja. 


\section{Anexo II}

Datos climáticos de las estaciones meterológicas más próximas a las localidades de Festuca patula. Las estaciones se presentan agrupadas por territorios. Para cada estación se indica su nombre, la provincia (abreviada), la precipitación media anual y la temperatura media anual de acuerdo con los datos disponibles en http://sig.marm.es/siga/

Cordilleras Béticas: Alcoy (A), $461 \mathrm{~mm}, 14,5^{\circ} \mathrm{C}$; Benaocaz (Ca) 1548,4 mm, 16,4 ${ }^{\circ} \mathrm{C}$; Grazalema (Ca) 1944,4 mm, $15,3{ }^{\circ} \mathrm{C}$; GrazalemaBenamaoma (Ca) $1170,7 \mathrm{~mm}$; Carcabuey (Co) $763 \mathrm{~mm}, 14,3^{\circ} \mathrm{C}$; Villaviciosa de Córdoba (Co) $856 \mathrm{~mm}$; Castril Las Hazadillas (Gr) $549 \mathrm{~mm}$, 13,2 ${ }^{\circ} \mathrm{C}$; Castril-Las Pocas (Gr) $471 \mathrm{~mm}, 13,7^{\circ} \mathrm{C}$; Castril-Nacimiento (Gr) $753 \mathrm{~mm}$; Cazorla-El Chorro (J) $963,8 \mathrm{~mm}$, Cazorla-Fuente El Oso (J) 1294,2 mm; Cazorla-ICONA (J) 740,3 mm, $14,8^{\circ} \mathrm{C}$; Cazorla Vadillo (J) 1.127,6 mm, $11,7^{\circ} \mathrm{C}$; Huelma-Ermita Fuensanta (J) $751 \mathrm{~mm}$; La Iruela (J) 764,4 mm; Segura de La Sierra-La Laguna (J) 960,9 mm; Alfarnate (Ma) $1032,5 \mathrm{~mm}, 13{ }^{\circ} \mathrm{C}$; Puerto de los Alazores-Venta Nueva (Ma) 862,8 mm; Ronda-LIFA (Ma) 832,6 mm. Las Villuercas-Monfra- güe: Alía (Cc) $784 \mathrm{~mm}$; Berzocana $(\mathrm{Cc}) 1.030 \mathrm{~mm}, 15,5^{\circ} \mathrm{C}$; Cañameroel Pinar (Cc) $854 \mathrm{~mm}, 15^{\circ} \mathrm{C}$; Garvín de la Jara-El Planchón (Cc) 726 $\mathrm{mm}, 14,3^{\circ} \mathrm{C}$; Guadalupe-Monasterio $(\mathrm{Cc}) 879 \mathrm{~mm}, 15,4^{\circ} \mathrm{C}$; Torrejón el Rubio-El Salto (Cc) $601 \mathrm{~mm}, 17,7^{\circ} \mathrm{C}$. Montes de Toledo: La Puebla de Don Rodrigo (CR) $588 \mathrm{~mm}, 15,2^{\circ} \mathrm{C}$; Navas de Estena (CR) $762 \mathrm{~mm}$, $14,4{ }^{\circ} \mathrm{C}$; Los Navalucillos (To) $518 \mathrm{~mm}, 14,4^{\circ} \mathrm{C}$; Retuerta de Bullaque (To) $610 \mathrm{~mm}, 13,3^{\circ} \mathrm{C}$. Sierra Morena: Las Navas de la Concepción (Co) $738 \mathrm{~mm}, 15,6^{\circ} \mathrm{C}$; Fuencaliente (CR) $747 \mathrm{~mm}$; Solana del Pino (Cr) 570 $\mathrm{mm}, 14,6{ }^{\circ} \mathrm{C}$; Santa Elena-Valdeazores (J) $857 \mathrm{~mm}, 15,3{ }^{\circ} \mathrm{C}$. Sistema Central: Las Casas del Conde (Sa) $983,5 \mathrm{~mm}, 14,5^{\circ} \mathrm{C}$; Sequeros (Sa) $1.111,4 \mathrm{~mm}, 13,9^{\circ} \mathrm{C}$; Sotoserrano (Sa) $1.118,5 \mathrm{~mm}, 14,7^{\circ} \mathrm{C}$. 